\title{
Alignment of Non-Overlapping Sequences
}

\author{
Yaron Caspi Michal Irani \\ Dept. of Computer Science and Applied Math \\ The Weizmann Institute of Science \\ 76100 Rehovot, Israel
}

This paper shows how two image sequences that have no spatial overlap between their fields of view can be aligned both in time and in space. Such alignment is possible when the two cameras are attached closely together and are moved jointly in space. The common motion induces "similar" changes over time within the two sequences. This correlated temporal behavior, is used to recover the spatial and temporal transformations between the two sequences. The requirement of "coherent appearance" in standard image alignment techniques is therefore replaced by "coherent temporal behavior", which is often easier to satisfy.

This approach to alignment can be used not only for aligning non-overlapping sequences, but also for handling other cases that are inherently difficult for standard image alignment techniques. We demonstrate applications of this approach to three real-world problems: (i) alignment of non-overlapping sequences for generating wide-screen movies, (ii) alignment of images (sequences) obtained at significantly different zooms, for surveillance applications, and, (iii) multi-sensor image alignment for multi-sensor fusion.

\section{Introduction}

The problem of image alignment (or registration) has been extensively researched, and successful approaches have been developed for solving this problem. Some of these approaches are based on matching extracted local image features. Other approaches are based on directly matching image intensities. A review of some of these methods can be found in [19] and [13]. However, all these approaches share one basic assumption: that there is sufficient overlap between the two images to allow extraction of common image properties, namely, that there is sufficient "similarity" between the two images ("Similarity" of images is used here in the broadest sense. It could range from graylevel similarity, to feature similarity, to similarity of frequencies, and all the way to statistical similarity such as mutual information [21]).

In this paper the following question is addressed: Can two images be aligned when there is very little similarity between them, or even more extremely, when there is no spatial overlap at all between the two images? When dealing with individual images, the answer tends to be "No". However, this is not the case when dealing with image sequences. An image sequence contains much more information than any individual frame does. In particular, temporal changes (such as dynamic changes in the scene, or the induced image motion) are encoded between video frames, but do not appear in any individual frame. Such information can form a powerful cue for alignment of two (or more) sequences. Caspi and Irani [6] and Stein [18] have illustrated an applicability of such an approach for aligning two sequences based on common dynamic scene information. However, they assumed that the same temporal changes in the scene (e.g., moving objects) are visible to both video cameras, leading to the requirement that there must be significant overlap in the FOV's (fields-of-view) of the two cameras.

In this paper we show that when two cameras are attached closely to each other (so that their centers of projections are very close), and move jointly in space, then the induced frame-to-frame transformations within each sequence have correlated behavior across the two sequences. This is true even when the sequences have no spatial overlap. This correlated temporal behavior is used to recover both the spatial and temporal transformations between the two sequences.

Unlike carefully calibrated stereo-rigs [17], our approach does not require any prior internal or external camera calibration, nor any sophisticated hardware. Our approach bears resemblance to the approaches suggested by $[7,11,22]$ for auto-calibration of stereo-rigs. But unlike these methods, we do not require that the two cameras observe and match the same scene features, nor that their FOV's will overlap.

The need for "coherent appearance", which is a fundamental assumption in image alignment methods, is replaced here with the requirement of "coherent temporal behavior". A similar approach was used for "hand eye calibration" in robotics research e.g., [20,12]. Coherent temporal behavior is often easier to satisfy (e.g., by moving the two cameras jointly in space). Our approach is therefore useful not only in the case of non-overlapping sequences, but also in other cases which are inherently difficult for standard image alignment techniques.

This gives rise to a variety of real-world applications, in- 

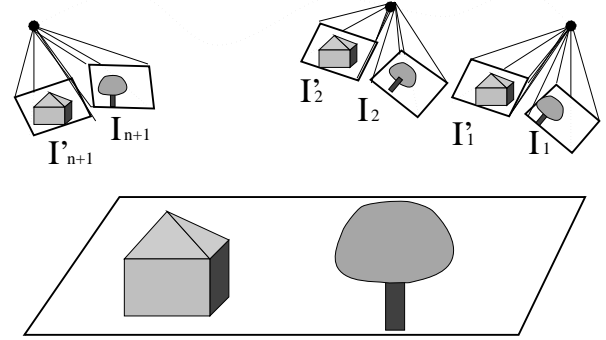

Figure 1: Two video cameras are attached to each other, so that they have the same center of projection, but non-overlapping fieldsof-view. The two cameras are moved jointly in space, producing two separate video sequences $I_{1}, \ldots, I_{n+1}$ and $I_{1}^{\prime}, \ldots, I_{n+1}^{\prime}$.

cluding: (i) Multi-sensor alignment for image fusion. This requires accurate alignment of images (sequences) obtained by sensors of different sensing modalities (such as InfraRed and visible light). Such images differ significantly in their appearance due to different sensor properties [21]. (ii) Alignment of images (sequences) obtained at different zooms. The problem here is that different image features are prominent at different image resolutions [8]. Alignment of a wide-FOV sequence with a narrow-FOV sequence is useful for detecting small zoomed-in objects in (or outside) a zoomed-out view of the scene. This can be useful in surveillance applications. (iii) Generation of wide-screen movies from multiple non-overlapping narrow FOV movies (such as in IMAX movies).

Our approach can handle such cases. Results are demonstrated in the paper on complex real-world sequences, as well as on manipulated sequences with ground truth.

\section{Problem Formulation}

We examine the case when two video cameras having (approximately) the same center of projection but different 3D orientation, move jointly in space (see Fig. 1). The fields of view of the two cameras do not necessarily overlap. The internal parameters of the two cameras are different and unknown, but fixed along the sequences. The external parameters relating the two cameras (i.e., the relative 3D orientation) are also unknown but fixed. Let $S=I_{1}, \ldots I_{n+1}$ and $S^{\prime}=I_{1}^{\prime}, \ldots, I_{m+1}^{\prime}$ be the two sequences of images recorded by the two cameras ${ }^{1}$. When temporal synchronization (e.g., time stamps) is not available, then $I_{i}$ and $I_{i}^{\prime}$ may not be corresponding frames in time. Our goal is to recover the transformation that aligns the two sequences both in time and in space. Note the term "alignment" here has a broader meaning than the usual one, as the sequences may not overlap in space, and may not be synchronized in time. Here we refer

\footnotetext{
${ }^{1}$ The subscript $i$ is used represents the frame time index, and the superscript prime is used to distinguish between the two sequences $S$ and $S$.
}

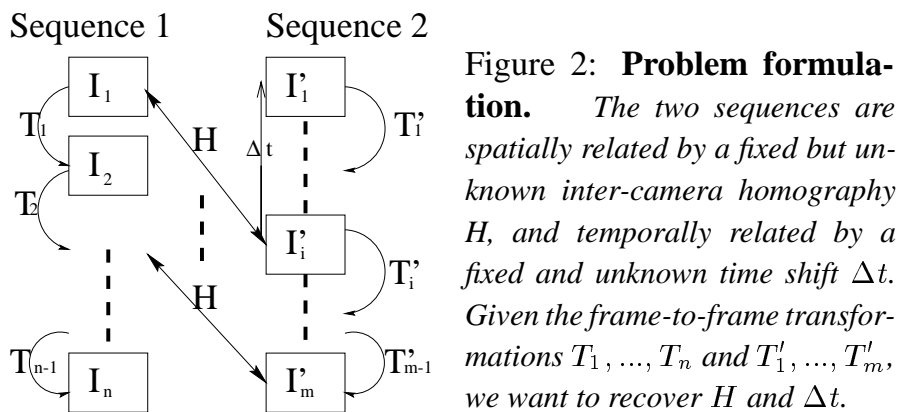

to alignment as displaying one sequence in the spatial coordinate system of the other sequence, and at the correct time shift, as if obtained by the other camera.

When the two cameras have the same center of projection (and differ only in their 3D orientation and their internal calibration parameters), then a simple fixed homography $H$ (a 2D projective transformation) describes the spatial transformation between temporally corresponding pairs of frames across the two sequences [10].

If there were enough common features (e.g., $p$ and $p^{\prime}$ ) between temporally corresponding frames (e.g., $I_{i}$ and $I_{i}^{\prime}$ ), then it would be easy to recover the inter-camera homography $H$, as each such pair of corresponding image points provides two linear constrains on $H: p^{\prime} \cong H p$. This, in fact, is how most image alignment techniques work [10]. However, this is not the case here. The two sequence do not share common features, because there is no spatial overlap between the two sequences. Instead, the homography $H$ is recovered from the induced frame-to-frame transformations within each sequence.

Let $T_{1}, \ldots T_{n}$ and $T_{1}^{\prime}, \ldots T_{m}^{\prime}$ be the sequences of frameto-frame transformations within the video sequences $S$ and $S^{\prime}$, respectively. $T_{i}$ is the transformation relating frame $I_{i}$ to $I_{i+1}$. These transformations can be either 2D parametric transformations (e.g., homographies or affine transformations) or 3D transformations/relations (e.g., fundamental matrices). We next show how we can recover the spatial transformation $H$ and the temporal shift $\Delta t$ between the two video sequences directly from the two sequences of transformations $T_{1}, \ldots T_{n}$ and $T_{1}^{\prime}, \ldots T_{m}^{\prime}$. The problem formulated above is illustrated in Fig. 2.

\section{Recovering Spatial Alignment Between Se- quences}

Let us first assume that the temporal synchronization is known. Such information is often available (e.g., from time stamps encoded in each of the two sequences). Section 4 shows how we can recover the temporal shift between the two sequences when that information is not available. Therefore, without loss of generality, it is assumed that $I_{i}$ 
and $I_{i}^{\prime}$ are corresponding frames in time in sequences $S$ and $S^{\prime}$, respectively. Two cases are examined: (i) The case when the scene is planar or distant from the cameras. We refer to these scenes as "2D scenes". In this case the frame-to-frame transformations $T_{i}$ can be modeled by homographies (Sec. 3.1). (ii) The case of a non-planar scene. We refer to these scenes as "3D scenes". In this case the frame-to-frame relation can be modeled by a fundamental matrix (Sec. 3.2).

\subsection{Planar or Distant (2D) Scenes}

When the scene is planar or distant from the cameras, or when the joint 3D translation of the two cameras is negligible relative to the distance of the scene, then the induced image motions within each sequence (i.e., $T_{1}, \ldots T_{n}$ and $T_{1}^{\prime}, \ldots T_{n}^{\prime}$ ) can be described by $2 \mathrm{D}$ parametric transformations [10]. $T_{i}$ thus denotes the homography between frame $I_{i}$ and $I_{i+1}$, represented by $3 \times 3$ non-singular matrices. We next show that temporally corresponding transformations $T_{i}$ and $T_{i}^{\prime}$ are also related by the fixed inter-camera homography $H$ (which relates frames $I_{i}$ and $I_{i}^{\prime}$ ).

Let $P$ be a 3D point in the planar (or the remote) scene. Denote by $p_{i}$ and $p_{i}^{\prime}$ its image coordinates in frames $I_{i}$ and $I_{i}^{\prime}$, respectively (the point $P$ need not to be visible in the frames, i.e., $P$ need not be within the FOV of the cameras). Let $p_{i+1}$ and $p_{i+1}^{\prime}$ be its image coordinates in frames $I_{i+1}$ and $I_{i+1}^{\prime}$, respectively. Then, $p_{i+1} \cong T_{i} p_{i}$ and $p_{i+1}^{\prime} \cong$ $T_{i}^{\prime} p_{i}^{\prime}$. Because the coordinates of the video sequences $S$ and $S^{\prime}$ are related by a fixed homography $H$, then: $p^{\prime} \cong H p$ and $p_{i+1}^{\prime} \cong H p_{i+1}$. Therefore:

$$
H T_{i} p_{i} \cong H p_{i+1} \cong p_{i+1}^{\prime} \cong T_{i}^{\prime} p_{i}^{\prime} \cong T_{i}^{\prime} H p_{i}
$$

Each $p_{i}$ could theoretically have a different scalar associated with the equality in Eq. (1). However, it is easy to show that because the relation in Eq. (1) holds for all points $p_{i}$, therefore all these scalars are equal, and hence:

$$
H T_{i} \cong T_{i}^{\prime} H
$$

Because $H$ is invertible, we may write $T_{i}^{\prime} \cong H T_{i} H^{-1}$, or

$$
T_{i}^{\prime}=s_{i} H T_{i} H^{-1}
$$

where $s_{i}$ is a (frame-dependent) scale factor. Eq. (3) is true for all frames (i.e., for any pair of corresponding transformations $T_{i}$ and $\left.T_{i}^{\prime}, i=1 . . n\right)$. Eq. (3) shows that there is a similarity relation ${ }^{2}$ (or conjugacy relation) between the two matrices $T_{i}$ and $T_{i}^{\prime}$ (up to a scale factor). A similar observation was made for case of hand-eye calibration (e.g., $[20,12])$, and for auto-calibration of a stereo-rig (e.g. [22]).

\footnotetext{
${ }^{2}$ A matrix $A$ is said to be "similar" to a matrix $B$ if there exists an invertible matrix $M$ such that $A=M B M^{-1}$. See [9]. The term "conjugate matrices" can be used as well.
}

Denote by eig $(A)=\left[\lambda_{1}, \lambda_{2}, \lambda_{3}\right]^{t}$ a $3 \times 1$ vector containing the eigenvalues of a $3 \times 3$ matrix $A$ (in decreasing order). Then it is known ([9] pp. 898.) that: (i) If $A$ and $B$ are similar matrices, then they have the same eigenvalues: $e i g(A)=e i g(B)$, and, (ii) The eigenvalues of a scaled matrix are scaled: $\operatorname{eig}(s A)=s(\operatorname{eig}(A))$. Using these two facts and Eq. (3) we obtain:

$$
\operatorname{eig}\left(T_{i}^{\prime}\right)=s_{i} \operatorname{eig}\left(T_{i}\right)
$$

where $s_{i}$ is the scale factor defined by Eq. (3). Eq. (4) implies that $e i g\left(T_{i}\right)$ and $e i g\left(T_{i}^{\prime}\right)$ are "parallel". This gives rise to a measure of similarity between two matrices $T_{i}$ and $T_{i}^{\prime}$ :

$$
\operatorname{sim}\left(T_{i}, T_{i}^{\prime}\right)=\frac{\operatorname{eig}\left(T_{i}\right)^{t} \operatorname{eig}\left(T_{i}^{\prime}\right)}{\left\|\operatorname{eig}\left(T_{i}\right)\right\|\left\|\operatorname{eig}\left(T_{i}^{\prime}\right)\right\|},
$$

where $\|\cdot\|$ is the vector norm. For real valued eigenvalues, Eq. (5) provides the cosine of the angle between the two vectors eig $\left(T_{i}\right)$ and $e i g\left(T_{i}^{\prime}\right)$. This property will be used later for obtaining the temporal synchronization (Section 4). This measure is also used for outlier rejection of bad frame-toframe transformation pairs, $T_{i}$ and $T_{i}^{\prime}$. The remainder of this section explains how the fixed inter-camera homography $\mathrm{H}$ is recovered from the list of frame-to-frame transformations $T_{1}, . . T_{n}$ and $T_{1}^{\prime}, . ., T_{n}^{\prime}$, and discusses uniqueness of the solution.

For each pair of temporally corresponding transformations $T_{i}$ and $T_{i}^{\prime}$ in sequences $S$ and $S^{\prime}$, we first compute their eigenvalues $\operatorname{eig}\left(T_{i}\right)$ and $\operatorname{eig}\left(T_{i}^{\prime}\right)$. The scale factor $s_{i}$ which relates them is then estimated from Eq. (4) using least squares minimization. (three equations one unknown). Once $s_{i}$ is estimated, Eq. (3) (or Eq. (2)) can be rewritten as:

$$
s_{i} H T_{i}-T_{i}^{\prime} H=0
$$

Eq. (6) is linear in the unknown components of $H$. Rearranging the components of $H$ in a $9 \times 1$ column vector $\vec{h}=\left[H_{11} H_{12} H_{13} H_{21} H_{22} H_{23} H_{31} H_{32} H_{33}\right]^{t}$, Eq. (6) can be rewritten as a set of linear equations in $\vec{h}$ :

$$
M_{i} \vec{h}=\overrightarrow{0}
$$

where $M_{i}$ is a $9 \times 9$ matrix defined by $T_{i}, T_{i}^{\prime}$ and $s_{i}$ :

$M_{i}=\left[\begin{array}{c|c|c}s_{i} T_{i}^{t}-T_{i_{11}}^{\prime} I & -T_{i_{12}}^{\prime} I & -T_{i_{13} I}^{\prime} I \\ \hline-T_{i_{21}}^{\prime} I & s_{i} T^{t}-T_{i_{22}}^{\prime} I & -T_{i_{23} I}^{\prime} I \\ \hline-T_{i_{31}}^{\prime} I & -T_{i_{32}}^{\prime} I & s_{i} T^{t}-T_{i_{33}}^{\prime} I\end{array}\right]_{9 \times 9}$

and $I$ is the $3 \times 3$ identity matrix.

Eq. (7) implies that each pair of corresponding transformations $T_{i}$ and $T_{i}^{\prime}$ contributes 9 linear constrains in the unknown homography $H$ (i.e., $\vec{h}$ ). It can be shown [5] that if $T_{i}$ (and hence also $T_{i}^{\prime}$ ) have 3 different eigenvalues, then $H$ can be determined by a single such pair of transformations 


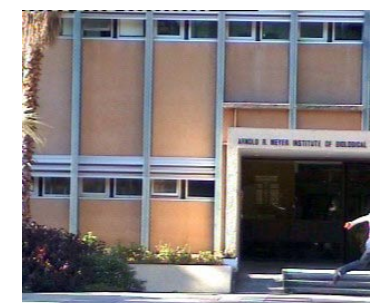

(a)

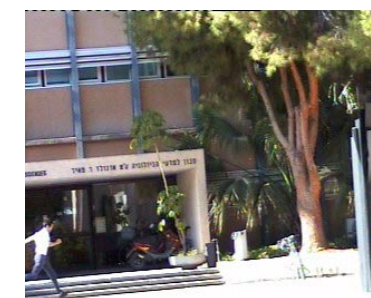

(b)

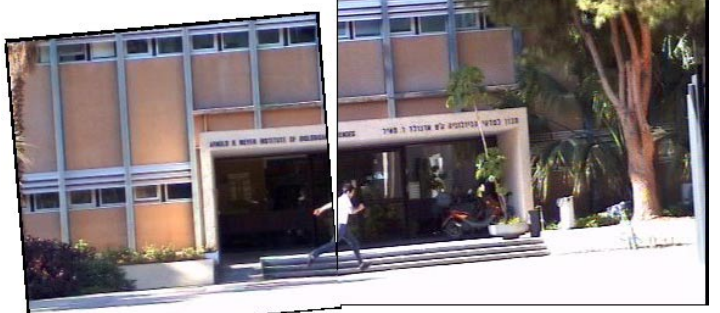

(c)

Figure 3: Alignment of non-overlapping sequences. (a) and $(b)$ are temporally corresponding frames from sequences $S$ and $S^{\prime}$. The correct time shift was automatically detected. (c) shows one frame in the combined sequence after spatiotemporal alignment. Note the accuracy of the spatial and temporal alignment of the running person. For full sequences see www.wisdom.weizmann.ac.il/NonOverlappingSeqs.

up to three degrees of freedom. Therefore, at least two such pairs of independent transformations are needed to uniquely determine the homography $H$ (up to a scale factor).

The constraints from all the transformations $T_{1}, . ., T_{n}$ and $T_{1}^{\prime}, . ., T_{n}^{\prime}$ can be combined into a single set of linear equations in $\vec{h}$ :

$$
A \vec{h}=\overrightarrow{0}
$$

where A is a $9 n \times 9$ matrix: $A=\left[\begin{array}{c}M_{1} \\ \vdots \\ M_{n}\end{array}\right]$. Eq. (8) is a homogeneous set of linear equations in $\vec{h}$, that can be solved in a variety of ways [3]. In particular, $\vec{h}$ may be recovered by computing the eigenvector which corresponds to the smallest eigenvalue of the matrix $A^{t} A$.

\subsection{D Scenes}

When the scene is neither planar nor distant, the relation between two consecutive frames of an uncalibrated camera is described by the fundamental matrix [10]. In this case the input to our algorithm is two sequences of fundamental matrices between successive frames, denoted by $F_{1}, \ldots F_{n}$ and $F_{1}^{\prime}, \ldots F_{n}^{\prime}$. Namely, if $p_{i} \in I_{i}$ and $p_{i+1} \in I_{i+1}$ are corresponding image points, then: $p_{i+1}^{t} F_{i} p_{i}=0$. Although the relations within each sequence are characterized by fundamental matrices, the inter-camera transformation remains a (a)

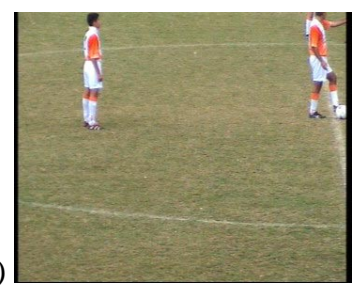

(b)

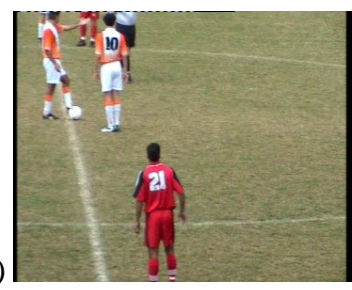

(c)

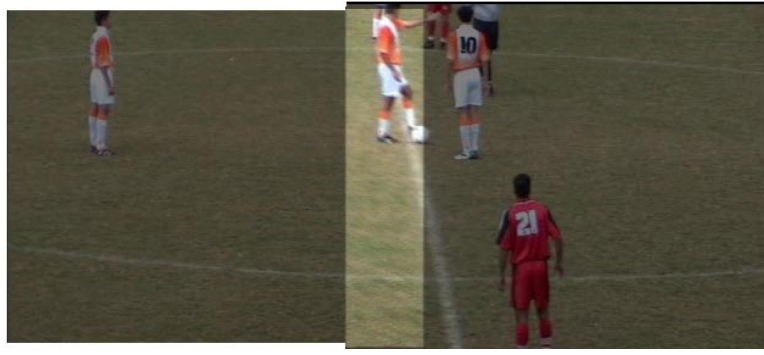

Figure 4: Alignment of non-overlapping sequences. (a) and $(b)$ are temporally corresponding frames from sequences $S$ and $S^{\prime}$. The correct time shift was automatically detected. (c) shows one frame in the combined sequence. Corresponding video frames were averaged after spatio-temporal alignment. The small overlapping area was not used in the estimation process, but only for verification (see text). Note the accuracy of the spatial and temporal alignment of the soccer player in the overlapping region. For full sequences see www.wisdom.weizmann.ac.il/NonOverlappingSeqs.

homography $H$. This is because the two cameras still share the same center of projection (Sec. 2).

Each fundamental matrix $F_{i}$ can be decomposed into a homography + epipole as follows [10]:

$$
F_{i}=\left[e_{i}\right]_{x} T_{i}
$$

where $e_{i}$ is the epipole relating frames $I_{i}$ and $I_{i+1}$, the matrix $T_{i}$ is the induced homography from $I_{i}$ to $I_{i+1}$ via any plane (real or virtual). $[\cdot]_{x}$ is the cross product matrix $\left([v]_{x} \vec{w}=\vec{v} \times \vec{w}\right)$.

The homographies, $T_{1}, \ldots, T_{n}$ and $T_{1}^{\prime}, \ldots, T_{n}^{\prime}$, and the epipoles $e_{1}, \ldots, e_{n}$ and $e_{1}^{\prime}, \ldots, e_{n}^{\prime}$, impose separate constraints on the inter-camera homography $H$. These constraints can be used separately or jointly to recover $H$.

(i) Homography-based constraints: The homographies $T_{1}, . ., T_{n}$ and $T_{1}^{\prime}, . ., T_{n}^{\prime}$ (extracted from the fundamental matrices $F_{1}, . ., F_{n}$ and $F_{1}^{\prime}, . ., F_{n}^{\prime}$, respectively), may correspond to different $3 \mathrm{D}$ planes. In order to apply the algorithm of Sec. 3.1 using these homographies, we need impose plane-consistency across the two sequences (to guarantee that temporally corresponding homographies correspond to the same plane in the 3D world). One possible way for imposing plane-consistency across (and within) the two sequences is by using the "Plane+Parallax" approach $[16,14]$. However, this approach requires that a real phys- 
ical planar surface be visible in all video frames. Alternatively, the "threading" method of [1] or other methods for computing consistent set of camera matrices (e.g., [2]), can impose plane-consistency within each sequence, even if no real physical plane is visible in any of the frames. Plane consistency across the two sequences can be guaranteed e.g., if [1] is initiated at frames which are known to simultaneously view the same real plane in both sequences. However, the two cameras can see different portions of the plane (allowing for non-overlapping FOVs), and need not see the plane at any of the other frames. This approach is therefore less restrictive than the Plane+Parallax approach.

(ii) Epipole-based constraints: The fundamental matrices $F_{1} . . F_{n}$ and $F_{1}^{\prime} . . F_{n}^{\prime}$ also provide a list of epipoles $e_{1}, \ldots, e_{n}$ and $e_{1}^{\prime}, \ldots, e_{n}^{\prime}$. These epipoles are uniquely defined (there is no issue of plane consistency here). Since the two cameras have the same center of projection, then for any frame $i: e_{i}^{\prime} \cong H e_{i}$, or more specifically:

$$
\left(e_{i}^{\prime}\right)_{x}=\frac{\left[h_{1} h_{2} h_{3}\right] e_{i}}{\left[h_{7} h_{8} h_{9}\right] e_{i}} \quad\left(e_{i}^{\prime}\right)_{y}=\frac{\left[h_{4} h_{5} h_{6}\right] e_{i}}{\left[h_{7} h_{8} h_{9}\right] e_{i}}
$$

Multiplying by the dominator and rearranging terms yields two new linear constrains on $H$ for every pair of corresponding epipoles $e_{i}$ and $e_{i}^{\prime}$ :

$$
\left[\begin{array}{ccc}
e_{i}{ }^{t} & \overrightarrow{0}^{t} & \left(e_{i}^{\prime}\right)_{x} e_{i}{ }^{t} \\
\overrightarrow{0}^{t} & e_{i}{ }^{t} & \left(e_{i}^{\prime}\right)_{y} e_{i}^{t}
\end{array}\right]_{2 \times 9} \vec{h}=0
$$

where $\overrightarrow{0}^{t}=[0,0,0]$. Every pair of temporally corresponding epipoles, $e_{i}$ and $e_{i}^{\prime}$, thus imposes two linear constraints on $H$. These $2 n$ constraints $(i=1, . ., n)$ can be added to the set of linear equations in Eq. (8) which are imposed by the homographies. Alternatively, the epipole-related constraints can be used alone to solve for $H$, thus avoiding the need to enforce plane-consistency on the homographies. Theoretically, four pairs of corresponding epipoles $e_{i}$ and $e_{i}^{\prime}$ are sufficient.

\section{Recovering Temporal Synchronization Be- tween Sequences}

So far we have assumed that the temporal synchronization between the two sequences is known and given. Namely, that frame $I_{i}$ in sequence $S$ corresponds to frame $I_{i}^{\prime}$ in sequence $S^{\prime}$, and therefore the transformation $T_{i}$ corresponds to transformation $T_{i}^{\prime}$. Such information is often available from time stamps. However, when such synchronization is not available, we can recover it. Given two unsynchronized sequences of transformations $T_{1}, \ldots T_{n}$ and $T_{1}^{\prime}, \ldots T_{m}^{\prime}$, we wish to recover the unknown temporal shift $\Delta t$ between them. Let $T_{i}$ and $T_{i+\Delta t}^{\prime}$ be temporally corresponding transformations (namely, they occurred at the same time instance). Then from Eq. (4) we know that they should satisfy $\operatorname{eig}\left(T_{i}\right) \| \operatorname{eig}\left(T_{i+\Delta t}^{\prime}\right)$ (i.e., the $3 \times 1$ vectors of eigenvalues should be parallel). In other words, the similarity measure $\operatorname{sim}\left(T_{t_{i}}, T_{t_{i}^{\prime}+\Delta t}^{\prime}\right)$ of Eq. (5) should equal 1 (corresponding to $\cos (0)$, i.e., an angle of $0^{\circ}$ between the two vectors). All pairs of corresponding transformations $T_{i}$ and $T_{i+\Delta t}^{\prime}$ must simultaneously satisfy this constraint for the correct time shift $\Delta t$. Therefore, we recover the unknown temporal time shift $\Delta t$ by maximizing the following objective function:

$$
S I M(\Delta t)=\sum_{i} \operatorname{sim}\left(T_{i}, T_{i+\Delta t}\right)^{2}
$$

The maximization is currently performed by an exhaustive search over a finite range of valid time shifts $\Delta t$. To address larger temporal shifts, we apply a hierarchical search. Coarser temporal levels are constructed by composing transformations to obtain fewer transformation between more distant frames.

The objective function of Eq. (11) can be generalized to handle sequences of different frame rates, such as sequences obtained by NTSC cameras ( $30 \mathrm{frame} / \mathrm{sec}$ ) vs. PAL cameras ( 25 frames/sec). The ratio between frames corresponding to equal time steps in the two sequences is $25: 30=5: 6$. Therefore, the objective function that should be maximized for an NTSC-PAL pair of sequences is:

$$
\operatorname{SIM}(\Delta t)=\sum_{i} \operatorname{sim}\left(T_{5 i}^{5(i+1)}, T^{\prime 6(i+1)+\Delta t}\right)^{2}
$$

Where $T_{i}^{j}$ is the transformation from frame $I_{i}$ to frame $I_{j}$. In our experiments, all sequences were obtained by PAL video cameras. Therefore only the case of equal framerate (Eq. (11)) was experimentally verified. We found this method to be very robust. It successfully recovered the temporal shift up to field (sub-frame) accuracy. Sub-field accuracy may be further recovered by interpolating the values of $\operatorname{SIM}(\Delta t)$ obtained at discrete time shifts.

\section{Applications}

This section illustrates the applicability of our method to solving some real-world problems, which are particularly difficult for standard image alignment techniques. These include: (i) Alignment of non-overlapping sequences for generation of wide-screen movies from multiple narrowscreen movies (such as in IMAX films), (ii) Alignment of sequences obtained at significantly different zooms (e.g., for surveillance applications), and (iii) Alignment of multisensor sequences for multi-sensor fusion. We show results of applying the method to complex real-world sequences. In addition, in order to empirically quantify the accuracy of 

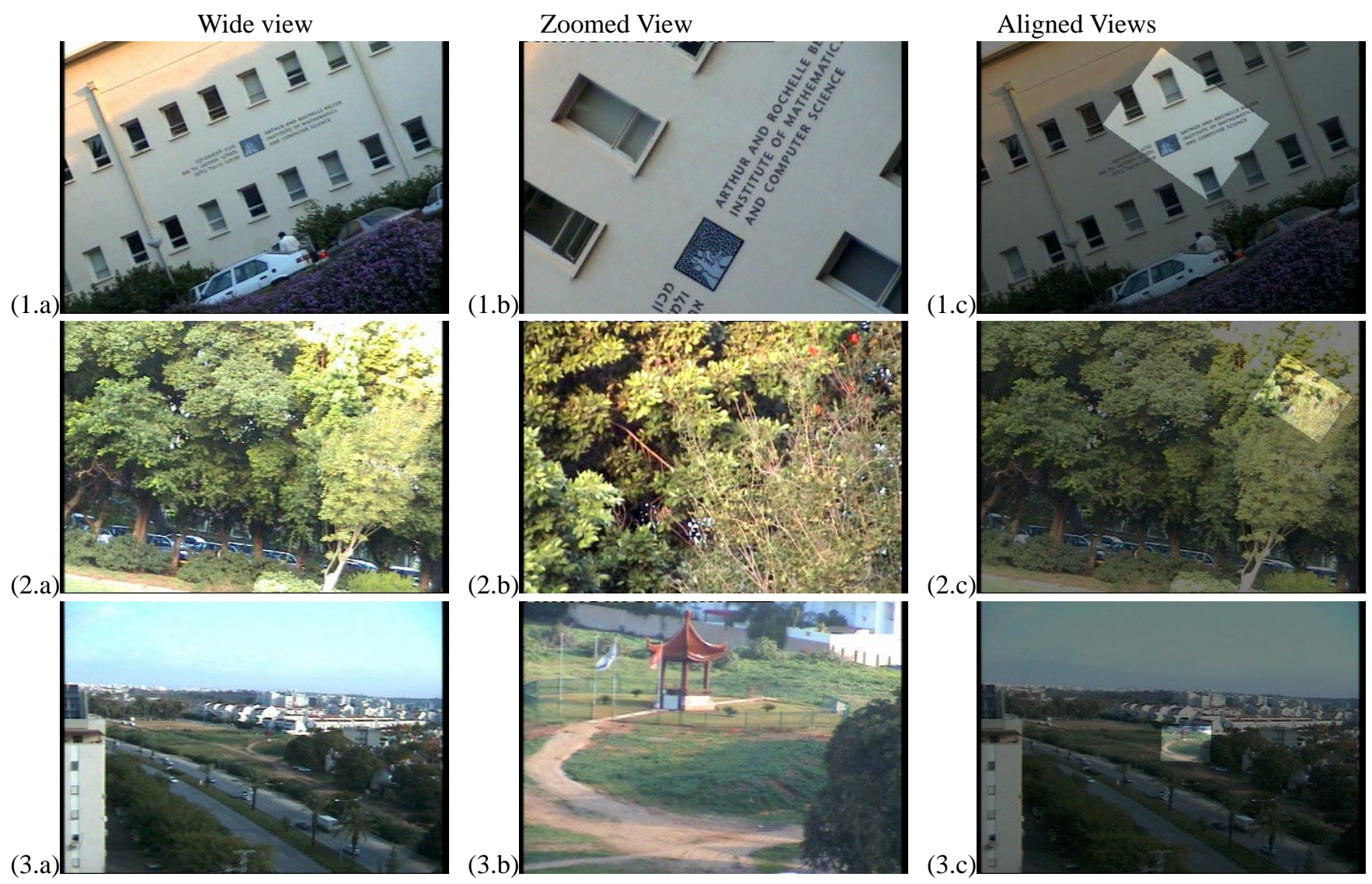

Figure 5: Finding zoomed region. This figure displays three different examples (one at each row), each one with different zoom factor. The left column (1.a, 2.a, 3.a) display one frame from each of the three wide-FOV sequences. The temporally corresponding frames from the corresponding narrow-FOV sequences are displayed in the center column. The correct time shift was automatically detected for each pair of narrow/wide FOV sequences. Each image on the right column shows super-position of corresponding frames of the two sequences after spatio-temporal alignment, displayed by color averaging. For full sequences see www.wisdom.weizmann.ac.il/NonOverlappingSeqs.

our method, we also applied it to pairs of sequences generated from a real sequence by warping it with known (ground truth) homographies. All sequences which we experimented with were captured by "of the shelf" consumer CCD cameras. The cameras were attached to each other, to minimize the distance between their centers of projections. The joint camera motion was performed manually (i.e., a person would manually hold and rotate the two attached cameras). No temporal synchronization tool was used.

The frame-to-frame input transformations within each sequence (homographies $T_{1} \ldots T_{n}$ and $T_{1}^{\prime} \ldots T_{n}^{\prime}$ ) were extracted using the method described in [15]. The input sequences were usually several seconds long to guaranty significant enough motion. The temporal time shift was recovered using the algorithm described in Sec. 4 up to field accuracy. Inaccurate frame-to-frame transformations $T_{i}$ were pruned out by using two outlier detection mechanisms. These are discussed in detail in [5]. Finally, the best thirty or so transformations were used in the estimation of the inter-camera homography $H$ (using the algorithm described in Sec. 3.1).

\subsection{Alignment of Non-Overlapping Sequences}

Fig. 3 shows an example of alignment of nonoverlapping sequences. The left camera is zoomed-in and rotated relative to the right camera. The correct spatio-temporal alignment can be seen in Fig. 3.c. Note the accurate alignment of the running person both in time and in space.

Our approach to sequence alignment can be used to generate wide-screen movies from two (or more) narrow fieldof-view movies (such as in IMAX movies). Such an example is shown in Fig. 4. To verify the accuracy of alignment (both in time and in space), we allowed for a very small overlap between the two sequences. However, this image region was not used in the estimation process, to imitate the case of truly non-overlapping sequences. The overlapping region was used only for display and verification purposes. Fig. 4.c shows the result of combining the two sequences (by averag- 
Visible

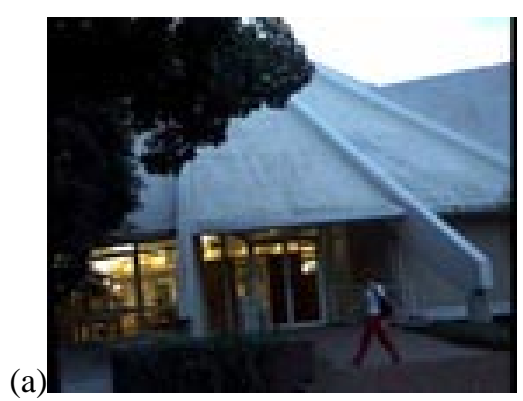

IR

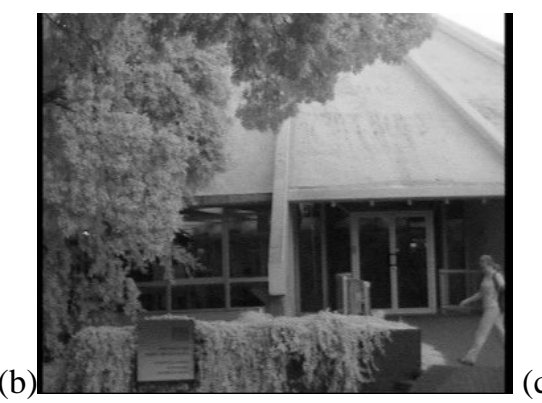

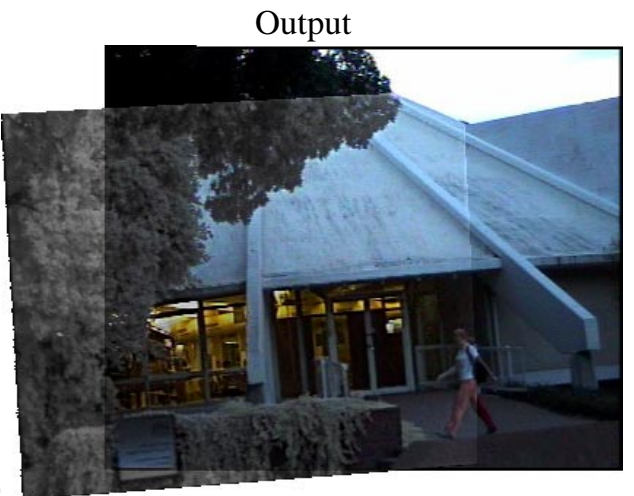

Figure 6: Multi-sensor Alignment. (a) and (b) are temporally corresponding frames from the visible-light and IR sequences, respectively (the temporal alignment was automatically detected). The inside of the building is visible only in the visible-light sequence, while the IR sequence captures the details outdoors (e.g., the dark trees, the sign, the bush). (c) shows the results of fusing the two sequences after spatio-temporal alignment. The fused sequence preserves the details from both sequences. Note the high accuracy of alignment (both in time and in space) of the walking lady. For more details see text. For full sequences see www.wisdom.weizmann.ac.il/NonOverlappingSeqs.

ing corresponding frames) after spatio-temporal alignment. Note the accurate spatial as well as temporal alignment of the soccer players in the averaged overlapping region.

In order to empirically verify the accuracy of our method, the real video sequence of Fig. 7 was split in the middle, producing two non-overlapping sequences of half-a-frame width each. The true (ground truth) homography in this case corresponds to a horizontal shift by the width of a frame (352 pixels). The frame-to-frame transformation $\left(T_{1} \ldots T_{n}\right.$ and $T_{1}^{\prime} \ldots T_{n}^{\prime}$ ) were estimated separately within each sequence using [15]. The temporal shift $(\Delta t=0)$ was recovered correctly from these transformations, and the "inter-camera" homography $H$ was recovered up to a misalignment error of less than 0.7 pixel over the entire image. See Table 1 for summary of the quantitative experimental results.

\subsection{Alignment of Sequences Obtained at Different Zooms}

Often in surveillance applications two cameras are used, one with a wide FOV (field-of-view) for observing large scene regions, and the other camera with a narrow FOV (zoomed-in) for detecting small objects. Matching two such images obtained at significantly different zooms is a difficult problem for standard image alignment methods, since the two images display different features which are prominent at the different resolutions. Our sequence alignment approach may be used for such scenarios. Fig. 5 shows three such examples. The results of the spatio-temporal alignment (right colunm of Fig. 5) are displayed in the form of averaging temporally corresponding frames after alignment according to the computed homography and the computed time shift. In the first example (top row of Fig. 5) the zoom difference between the two cameras was approximately 1:3. In the sec- ond example (second row) it was $\approx 1: 4$, and in the third example (bottom row) it was $\approx 1: 8$. Note the small red flowers in the zoomed view (Fig. 5.2.b). These can barely be seen in the corresponding low resolution wide-view frame (Fig. 5.2.a). The same holds for the Pagoda in Fig. 5.3.b

To empirically verify the accuracy of our method in the presence of large zooms and large rotations, we ran the algorithm on following three manipulated sequences with known (ground truth) manipulations: We warped the sequence of Fig. 7 once by a zoom factor of 2, once by a zoom factor of 4 , and once rotated it by $180^{\circ}$. The results are summarized in Table 1.

In each of these cases, the recovered homography was composed with the inverse of the ground-truth homography: $H_{\text {true }}^{-1} H_{\text {recovored }}$. Ideally, the composed homography should be the identity matrix. The errors reported in Table 1 are the maximal residual misalignment induced by the composed homography over the entire image.

\subsection{Multi-Sensor Alignment}

Images obtained by sensors of different modalities, e.g., IR (Infra-Red) and visible light, can vary significantly in their appearance. Features appearing in one image may not appear in the other, and visa versa. This poses a problem for image alignment methods. Our sequence alignment approach, however, does not require coherent appearance between the two sequences, and can therefore be applied to solve the problem. Fig. 6 shows an example of two such sequences, one captured by a near IR camera, while the other by a regular video (visible-light) camera. The scene was shot in twilight. In the sequence obtained by the regular video camera (Fig.6.(a)), the outdoor scene is barely visible, while the inside of the building is clearly visible. The 

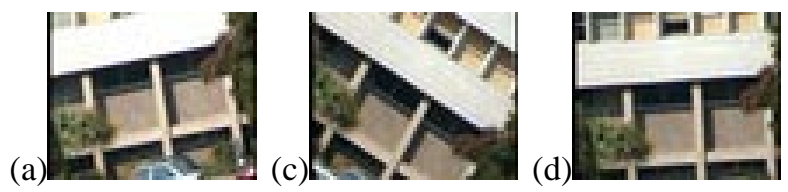

Figure 7: The sequence used for empirical evaluation. $(a, b, c)$ are three frames $(0,150,300)$ out of the original 300 frames. This sequence was used as the base sequence for the quantitative experiments summarized in Table 1.

IR camera, on the other hand, captures the outdoor scene in great detail, while the indoor part (illuminated by "cold" neon light) was invisible to the IR camera (Fig. 6.(b)). The result of the spatio-temporal alignment is illustrated by fusing temporally corresponding frames. The IR camera provides only intensity information, and was therefore fused only with the intensity (Y) component of the visible-light camera (using the image-fusion method of [4]). The chrome components (I and Q) of the visible-light camera supply the color information.

The reader is encouraged to view color sequences at www.wisdom.weizmann.ac.il/NonOverlappingSeqs.

\section{Conclusion}

This paper presents an approach for aligning two sequences (both in time and in space), even when there is no common spatial information between the sequences. This was made possible by replacing the need for "coherent appearance" (which is a fundamental requirement in standard images alignment techniques), with the requirement of "coherent temporal behavior", which is often easier to satisfy. We demonstrated applications of this approach to real-world problems, which are inherently difficult for regular image alignment techniques.

\section{Acknowledgment}

The authors would like to thank R. Basri and L. ZelnikManor for their helpful comments.

\section{References}

[1] S. Avidan and A. Shashua. Thereading fundamaental matrices. In European Conference on Computer Vision, pages 124-140, Freiburg, June 1998.

[2] P. A. Beardsley, P. H. S. Torr, and A. Zisserman. 3D model aquisition from extended image sequences. In Proc. 4th European Conference on Computer Vision, LNCS 1065, Cambridge, pages 683-695, 1996.

[3] A. Bjorck. Numerical Methodes for Least Squares Problems. SIAM, Philadelphia, 1996.

[4] P.R. Burt and R.J. Kolczynski. Enhanced image capture through fusion. In International Conference on Computer Vision, 1993.

[5] Y. Caspi and M. Irani. Alignment of non overlapping sequences. jornal version.

\begin{tabular}{|l|c|c|}
\hline $\begin{array}{c}\text { Applied } \\
\text { Transformation }\end{array}$ & $\begin{array}{c}\text { Recovered } \\
\text { Transformation }\end{array}$ & $\begin{array}{c}\text { Max Residual } \\
\text { Misalignment }\end{array}$ \\
\hline $\begin{array}{c}\text { Horizontal shift } \\
\text { of } 352 \text { pixels }\end{array}$ & $\begin{array}{c}\text { Horizontal shift } \\
\text { of } 351.6 \text { pixels }\end{array}$ & 0.7 pixels \\
\hline Zoom factor $=2$ & Zoom factor $=1.9992$ & 0.4 pixels \\
\hline Zoom factor $=4$ & Zoom factor $=4.0048$ & 0.4 pixels \\
\hline Rotation by $180^{\circ}$ & Rotation by $180.00^{\circ}$ & 0.01 pixels \\
\hline
\end{tabular}

Table 1: Quantitative results. This table summarizes the quantitative results with respect to ground truth. Each row corresponds to one experiment. In each experiment a real video sequence (Fig. 7) was warped ("manipulated") by a known homography, to generate a second sequence. The left column describes the type of spatial transformation applied to the sequence, the center column describes the recovered transformation, and the right column describes the residual error between the ground-truth homography and the recovered homography (measured in maximal residual misalignment in the image space). In all 4 cases the correct temporal shift was recovered accurately. See text for further details.

[6] Y. Caspi and M. Irani. A step towards sequence-to-sequence alignment. In IEEE Conference on Computer Vision and Pattern Recognition, pages 682-689, 2000.

[7] D. Demirdijian, A. Zisserman, and R. Horaud. Stereo autocalibration from one plane. In European Conference on Computer Vision, 2000.

[8] Y. Dufournaud, C. Schmid, and R. Horaud. Matching images with different resolutions. In IEEE Conference on Computer Vision and Pattern Recognition, pages 612-619, 2000.

[9] C. E.Pearson (ed.). Handbook of applied mathematics - Second Edition. Van Nostrand Reinhold Company, New York, 1983.

[10] R. Hartley and A. Zisserman. Multiple View Geometry in Computer Vision. Cambridge university press, Cambridge, 2000.

[11] R. Horaud and G. Csurka. reconstruction using motions of a stereo rig. In International Conference on Computer Vision, pages 96-103, 1998.

[12] R. Horaud and F. Dornaika. Hand-eye calibration. International Journal of Robotics Research, 14(3):195-210, June 1995.

[13] M. Irani and P. Anandan. About direct methods. In Vision Algorithms Workshop, pages 267-277, Corfu, 1999.

[14] M. Irani, P. Anandan, and D. Weinshall. From reference frames to reference planes: Multi-view parallax geometry and applications. In European Conference on Computer Vision, Freiburg, June 1998.

[15] M. Irani, B. Rousso, and S. Peleg. Computing occluding and transparent motions. International Journal of Computer Vision, 12(1):5-16, January 1994.

[16] R. Kumar, P. Anandan, and K. Hanna. Direct recovery of shape from multiple views: A parallax based approach. In International Conference on Pattern Recognition, 1994.

[17] C.C. Slama. Manual of Photogrammetry. American Society of Photogrammetry and Remote Sensing, 1980.

[18] G. P. Stein. Tracking from multiple view points: Self-calibration of space and time. In DARPA IU Workshop, pages 1037-1042, 1998.

[19] P.H.S. Torr and A. Zisserman. Feature based methods for structure and motion estimation. In Vision Algorithms Workshop, 1999.

[20] R. Y. Tsai and R. K. Lenz. A new technique for full autonomous and efficient $3 \mathrm{~d}$ robotics hand/eye calibration. IEEE Journal of Robotics and Automation, 5(3):345-358, June 1989.

[21] P. Viola and W. Wells III. Alignment by maximization of mutual information. In International Conference on Computer Vision, pages 16-23, 1995.

[22] A. Zisserman, P.A. Beardsley, and I.D. Reid. Metric calibration of a stereo rig. In Workshop on Representations of Visual Scenes, 1995. 